\title{
Research on College English Reading Teaching Model based on the Psychological Linguistics
}

\author{
Ling Xiao \\ Changsha Medical University, Changsha, Hunan, 410219
}

Keywords: College English Reading, Psychological Linguistics, Teaching Model

\begin{abstract}
For a long time, college English teachers have invested a lot of energy to study the college English teaching mode, so as to improve the college English teaching effect and students' reading ability. Psycholinguistics not only provides an important theoretical basis for discourse comprehension, but also becomes a strong backing for college English teachers' reading teaching research. This paper analyzes the psychological process of reading and puts forward a new college English reading teaching model based on psycholinguistic theory.
\end{abstract}

\section{Introduction}

English reading classroom teaching has long played an important role in college English teaching and therefore, college English teachers have been constantly discussing the most reasonable reading teaching model. Linguistic theory is the basis of their research, and psychological linguistics theory based on the in-depth study is worthy of attention, from the perspective of reading the psychological behavior of reading, can make more college English teachers faster and better To establish the best mode of college English reading teaching.

First, the psycholinguistic theory of reading interpretation of the linguist Goodman (1976) pointed out: "Reading is a process of choice, it involves the reader from the visual input selected from the minimum language clues to make tentative judgment This judgment is either read or abandoned or improved. "Goodman also divided the mental process of reading into" experience "," prediction "," verification "and" affirmation or amendment ". These processes form a cycle of mutual understanding, digestion and expression. Goodman from the perspective of psycholinguistics shows that reading is a kind of positive psychological activity, which is the process of mutual communication between the reader and the author. The reader uses the language knowledge and reading experience and skills to provide the text information And the meaning of choice, reasoning, to achieve the final understanding.

Clark and Silbertein (1977) summarize the meaning of this reading pattern derived from psycholinguistics. They further point out that reading is a positive understanding of the process, and thus need to teach students "top-down" reading skills. From the predictive judgment, from the context of speculation, from the article to infer, quickly browse to understand the overall content of the article.

Carrel and Einsterhold (1983) explored the "top-down" reading model, which showed that the content and number of articles read by second language students was an important factor in the development of top-down skills.

Compared with these studies, Coady (1979) proposed a relatively complete model. According to this model, he argues that the understanding of reading material for English as a foreign language (EFL) and English as a second language (ESL) depends on background knowledge, conceptual abilities and process strategies Interaction, the concept of ability in this model refers to the reader's intrinsic psychological factors, it includes both intellectual and non-intellectual ability. The handling strategy refers to the reader's ability to read at all levels of the discourse, as well as many common language skills. However, little remains on the role of background knowledge.

The idea of schema theory emphasizes the role of background knowledge in reading comprehension, and provides some new ideas for reading research. According to this theory, the 
discourse provides clues for the reader to use their previously acquired knowledge (background knowledge) and to form a knowledge structure to trace or conceive the meaning (Rumelhart, 1980). The idea of schema theory also points out that understanding an article depends not only on a person's language knowledge, it is the process of the reader's background knowledge interacting with the content of the article. Concept schemas are divided into formal schema and content schema.

Barrett and Niles have done a further study of the mental activity process of reading. Barrett will read the process of understanding is divided into five steps: (1) the phrase, sentence analysis and understanding of the process; (2) information re-organization process; (3) reasoning process; (4) from the knowledge base call known The process of knowledge; (5) the assimilation of the process of thinking. Compared with Barrett, Niles's research suggests that readers must strive to: (1) find and understand the logical relationship of reading materials; (2) establish specific reading purposes; (3) use the old knowledge to deal with reading encountered Of the new content (Lv Shengtong, Jiang Ju, 1997).

From the above analysis we can see that reading is not only the process of obtaining information, but also the process of interpreting information. The reader's understanding of reading material relies on the interaction of background knowledge, conceptual competence, and processing strategies.

\section{Psychology and Reading}

"Language comprehension" is a topic of concentrated discussion in psycholinguistics. In short, it is about how people understand the language, including verbal perception, lexical extraction, sentence processing and discourse comprehension. And reading is the four processes to carry out the comprehensive use of the embodiment.

In the study of language perception conditions, "context" is the most important condition that affects speech perception. Another area of language perception research is the study of "written language understanding", which involves "visual induction", "letter recognition", "word meaning" and "information memory" and "information organization" and other aspects of exploration. In the research of "lexical extraction", the exploration mainly focuses on four aspects: "the basic elements of the word", "the research method of the psychological vocabulary", "the factors that affect the extraction and organization of the vocabulary" and the "vocabulary extraction model". Marslen and Wilson argue that when a person hears a word, all the words of the word will be activated. No matter what kind of theory, it is difficult to apply to the interpretation of the language to understand the complex process of all, but each "model" emphasizes the "activation" theory.

Sentence comprehension is an important part of the study of language comprehension. Psycholinguists believe that people are able to understand the language, because the information receiver and the information output of the minds of the common grammar rules or "agreement." However, the study of sentence comprehension is not so simple, it also involves the surface structure of the sentence and the deep structure of the problem, only to A said "It's too warm here." For example, if B from the sentence to understand the literal meaning, He is likely to not understand the true meaning of $\mathrm{A}$. Therefore, the syntactic processing is the second problem that psycholinguistics explores in language comprehension. The study of syntactic processing involves four issues: the relationship between "surface structure and deep structure", "the relationship between language ability and pragmatic competence", "decomposition of sentence structure" and "processing of clause"

Explore how people through the syntax processing to understand the compound sentence and its meaning. However, to correctly understand the sentence, sentence memory is also an important part. "Discourse comprehension" in the study, basically can be summarized as three aspects, namely, discourse coherence and understanding of the strategy of research, discourse memory research and discourse processing. In the context of coherent research, the "anaphora" and "cataphor", presented by Halliday and Hasan, have become a major theme of discourse coherence. But whether it is "on the finger", or "under the finger", are talking closely linked to the relationship between the text and 
coherent. In the study of discourse comprehension strategy, the "known or unknown strategy", "direct matching strategy", "bridging strategy" and "reviewing known information strategy" of discourse information become important clues to discuss discourse comprehension. With regard to the study of discourse memory, the "organizational planning framework" of the human cognitive system, presented by Bartlett, still affects the study of discourse memory in psycho-linguistics today. He believes that memory is active and Creativity is planned. In terms of discourse memory, "proposition" and "reasoning" become the center of research. Psycholinguists believe that not only the identification of the topic and the combination of sentences are closely related to the discourse memory, but the discourse comprehension also contributes to the discourse memory. In the research of discourse processing, the "discourse processing model" has become the central content of the study of discourse comprehension, such as "context model", "connection model", "construction and combination model", etc., from all angles the process of discourse comprehension.

\section{The Enlightenment of Psycholinguistics to Reading Teaching}

In the process of reading teaching, teachers cannot be too much explanation and other language activities to replace the students to read their own psychological process. The simple teacher explained that the students read the teaching mode of teaching so that students cannot put more opportunities to practice reading the psycholinguistic process, so contrary to the law of reading the psychological activity, the students' foreign language reading ability is difficult to improve. Therefore, they must go through the process of "free reading stage" to "free reading stage". In this process, teachers must give students enough time to read, at the same time not only pay attention to intensive teaching, but also cannot ignore the extensive reading and fast reading training, students have read, read, read, review Ability to create a language for students to meet the psychological linguistic reading environment, so that students really experience the process of reading the psychological language activities.

Language and culture are very close and the language cannot exist from the culture, cultural background knowledge is an important factor affecting reading comprehension. Language reflects the characteristics of a nation, it not only contains the nation's historical and cultural background, but also contains the nation's view of life, way of life and way of thinking. The cultural context in which a person depends on growth will directly affect what he says and what to say and explain to each other. If students lack the knowledge of these backgrounds, it will certainly affect students' understanding of reading materials, and sometimes even misunderstandings. In the teaching of reading, the teacher must first explain the background of the cultural background and the background information presented in the text, let the students know how the native language user of the language looks at things and how Observe the world, let the students understand how the mother tongue users use their language to reflect their social thoughts, habits, behavior, clear understanding of cultural barriers in understanding, to understand the historical background, to improve students to read comprehension accuracy. In the process of reading, the context for the text of the text is a clue to the understanding of the comprehensive using of reading skills plays a vital role. The creation of context refers to finding out the context of the various languages on which the comprehension of the chapter depends, and grasping the new semantic link network in the chapter. The semantic relations within the chapter are embodied in the semantic relations between the paragraphs and articles, the paragraphs and the paragraphs, the sentences and the center of the sentences, sentences and sentences, words and words. Teachers through classroom teaching, and as an example to guide students to use this context of the construction procedures used in the reading and learning inside and outside the class, for example, if the students can grasp the information according to the reading of the information and the word before and after the suffix to guess the meaning This reading skills, then it will not be because of the word barrier to make the content of reading fragmented, unable to understand. In the context of the use of a variety of different reading skills can achieve access to different information for reading purposes. 


\section{Conclusion}

Psycholinguistics provides an important starting point for the study of discourse comprehension and analysis from the theoretical point of view, and gives an important inspiration to the practice of college English reading teaching. I believe that there will be more college English teachers to join the study of psychological linguistics on the impact of reading teaching team.

\section{Acknowledgement}

The Guidance of Categorization and Non Categorization to the Design of Graded Teaching - A Case Study of Directional and Regular Classes in Changsha Medical University. Number: Changsha Medical University Teaching, (2015) 14-22.

\section{References}

[1] Ye Changqing. Study on the Cultivation of Critical Reading Mode in the Basic Stage of College English Majors [J]. Journal of Changjiang Normal University, 2012 (02)

[2] Yang Gang. Discussion on reading mode and ability training [J]. Journal of Beijing Second International Studies University, 1997 (02)

[3] Wang Bo Ran, Zhao Wen. Study on the correlation between English learners' reading patterns and their reading ability [J]. Journal of Northeastern University, 2014 (03)

[4] Ni Jincheng. Interactive reading mode and its empirical study [J]. Journal of Xi'an International Studies University, 2014 (03)

[5] Li Yang. Discussion on the Construction of Relevance Reading Mode [J]. Chinese Language and Literature, 2013 (07) 